\title{
Antrochoanal Polyp in an Unusual Case of Follicular Ameloblastoma of Maxilla: A Case Report
}

\author{
Anuja S Kulkarni ${ }^{1}$, Mrunal Kesari ${ }^{2}$, Yoganand Patil ${ }^{3}$
}

\begin{abstract}
Follicular ameloblastoma of maxilla is a well-known rare clinical entity known to be originated from odontogenic cells. Although ameloblastoma is developed from dental epithelial remnants, the presence of ameloblastoma in maxilla with epicenter from maxillary antrum and not at all from alveolar process is a rare phenomenon. Here we report a rare presentation of this tumor as antrochoanal polyp with epicenter in maxillary antrum in a 45-year-old lady who presented with unusual complaints of dry cough with a history of 6 months along with hypothyroidism.

Keywords: Antrochoanal polyp, Follicular ameloblastoma, Maxilla, Maxillary antrum, Odontogenic tumor.

International Journal of Head and Neck Surgery (2021): 10.5005/jp-journals-10001-1441
\end{abstract}

\section{Case Description}

A 45-year-old lady was presented to us with complaints of dry cough with a history of over 6 months. Besides this, the patient also had symptoms suggestive of hypothyroidism such as weight gain, lethargy, and increased daytime somnolence. The patient had a family history of bronchial asthma.

Clinical examination revealed right-sided nasal polyp, which was pale, painless, and did not bleed on touch.

The following specific investigations were done:

- Thyroid function test

- Diagnostic nasal endoscopy

- Contrast-enhanced computed tomography (CECT) paranasal sinuses (CECT PNS)

Thyroid function test confirmed hypothyroidism. Diagnostic nasal endoscopy revealed right-sided antrochoanal polyp (Fig. 1). Contrast-enhanced computed tomography PNS revealed polyp arising from right maxillary sinus extending to posterior choana suggestive of right antrochoanal polyp (Fig. 2).

Patient received treatment for hypothyroidism. Subsequently, she underwent functional endoscopic sinus surgery for excision of right antrochoanal polyp. During the surgery, the polyp was removed completely and the specimen was sent for histopathological examination. Histopatholoical examination revealed odontogenic epithelial islands in plexiform and reticular pattern. Cells show reverse polarization, stellate reticulumlike cells suggestive of follicular ameloblastoma (Fig. 3). On immunohistochemistry, the patient was positive for CK19, CK14 in dentine epithelium, and for calretinin in stellate reticulinlike cells (Fig. 4).

Subsequent to this, the patient was subjected to further evaluation in view of the final diagnosis. The following special investigations were done subsequently:

- Magnetic resonance imaging (MRI) of the paranasal sinuses and neck

- High-resolution computed tomography (HRCT) thorax

- Serum parathormone (Sr PTH)

- Vitamin D3 and calcium profile
${ }^{1}$ Department of ENT and Head-Neck Surgery, Jagjivan Ram Hospital, Mumbai, Maharashtra, India

2,3 Department of Pathology, Jagjivan Ram Hospital, Mumbai, Maharashtra, India

Corresponding Author: Anuja S Kulkarni, Department of ENT and Head-Neck Surgery, Jagjivan Ram Hospital, Mumbai, Maharashtra, India, Phone: +91 9819464115, e-mail: dr.anujakulkarni@gmail.com

How to cite this article: Kulkarni AS, Kesari M, Patil Y. Antrochoanal Polyp in an Unusual Case of Follicular Ameloblastoma of Maxilla: A Case Report. Int J Head Neck Surg 2021;12(3):114-118.

Source of support: Nil

Conflict of interest: None

Magnetic resonance imaging of paranasal sinuses revealed residual soft tissue mass in the right maxillary sinus, coming onto the anterior, inferior, and posterolateral wall of right maxillary sinus. Besides, this also revealed blockage of sinus drainage pathways of ipsilateral ethmoids and frontal and sphenoid sinuses with resultant retention of fluid in these sinuses (Fig. 5).

High-resolution computed tomography thorax revealed no abnormality. Serum parathormone levels were normal. Serum vitamin $D$ and calcium levels were within normal range.

Subsequently, the patient was planned for right total maxillectomy. Right-sided total maxillectomy was performed (Fig. 6A). Ipsilateral frontal, ethmoid, and sphenoid sinus clearance was done. Sphenoethmoid recess was cleared. Pterygoid base clearance was done. Reconstruction was done by using fascia lata sling for orbital support and by split thickness skin graft for lining the cavity followed by an obturator. [Clinical photograph of patient at one(1) week post operatively depicting healing cavity (Fig. 7)]

Specimen was removed by "en-bloc" resection (Fig. 6B), and was sent for histopathological examination, which confirmed complete clearance of disease and diagnosis of follicular ameloblastoma (Figs 6B and C).

As per histological and radiological correlation, the epicenter of the disease was in the maxillary bone; anterior, medial, and posterior walls of maxilla; in the maxillary antrum; and not at all in alveolar process. Follow-up examination at 6 weeks revealed no evidence of any disease.

() The Author(s). 2021 Open Access This article is distributed under the terms of the Creative Commons Attribution 4.0 International License (https://creativecommons. org/licenses/by-nc/4.0/), which permits unrestricted use, distribution, and non-commercial reproduction in any medium, provided you give appropriate credit to the original author(s) and the source, provide a link to the Creative Commons license, and indicate if changes were made. The Creative Commons Public Domain Dedication waiver (http://creativecommons.org/publicdomain/zero/1.0/) applies to the data made available in this article, unless otherwise stated. 
Patient had uneventful recovery and was asymptomatic at 1 year follow-up.

\section{Discussion}

Ameloblastoma was first described by Cusack 1827. 'Etymologically, the word "ameloblastoma" was derived from the the old French word "amel," which means enamel, and the Greek word "blastos," meaning germ or bud. ${ }^{2,3}$ It is a rare, benign, slow-growing but locally invasive neoplasm of odontogenic origin involving the mandible (80\%) and maxilla (20\%). ${ }^{4,5}$ Most common presentation is painless swelling of the mandible or maxilla. ${ }^{6}$ Pain is uncommon and occasionally occur posthemorrhage following a fine needle aspiration (FNA). ${ }^{7}$ Pain with rapid growth represents the rare malignant ameloblastoma. Tooth displacement and root resorption are rare. Paresthesias are rare except in cases of perineural invasion.

Evaluation requires imaging and tissue diagnosis. On imaging, X-ray pantomography depicts a lytic lesion scalloped margin in case of unicystic lesions and soap bubble appearance in multicystic lesions. Computed tomography (CT) scan aids in determining the extent in well-defined radiolucent uni/multilocular

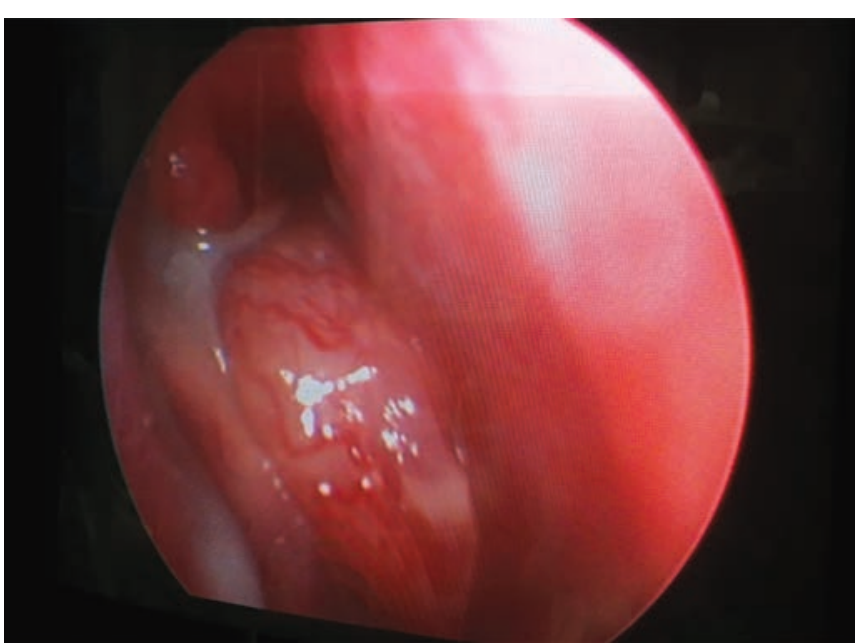

Fig. 1: Clinical photograph presentation as antrochoanal polyp expansile lesions. In addition, it gives useful information about the extent of cortical destruction. ${ }^{8}$ Magnetic resonance imaging provides potentially more complete information than $\mathrm{CT}$ about soft tissue extension and marrow extension beyond the lytic bone cavity. It is particularly useful for ameloblastomas arising from the maxilla, as it helps to characterize extension to the orbit, paranasal sinuses, and skull base. Magnetic resonance imaging is also considered for diagnosing desmoplastic ameloblastomas because they have poorly defined soft tissue borders and are often misdiagnosed as a fibro-osseous lesion. Positron emission tomography (PET) - CT is generally reserved for metastatic ameloblastoma, where it may aid with staging of the distant metastasis. ${ }^{8,9}$

Tissue diagnosis aids in deciding appropriate treatment modality. Fine Needle Aspiration Cytology (FNAC) from window of cortical erosion as identified by imaging or from the dental socket may provide useful information on tissue diagnosis. However, in case of cystic lesions core biopsy may have to be taken in order to obtain tissue diagnosis. This helps to rule out other similar lesions and conditions such as osteomyelitis, cystic fibrous dysplasia, giant cell tumor, ossifying fibroma, multiple myeloma, and rare sarcomas. ${ }^{9} \mathrm{WHO}$ (2005) classification for ameloblastomas includes four subtypes: ${ }^{4}$

- The solid/multicystic $91 \%$ unicystic type $6 \%$, the extra osseous ameloblastoma $2 \%$, and the desmoplastic type $1 \%$.

- Elzay, Slootweg, and Muller coined two types: metastatic ameloblastoma and ameloblastic carcinoma, comprising $2 \%$ of all ameloblastomas.

- Metastatic ameloblastoma has well-differentiated benign histology similar to the solid/multicystic type at the primary site and at remote site.

- In ameloblastic carcinoma, malignant histologic features are increased or has abnormal mitoses and cytological atypia.

Most common site for regional metastasis include neck nodes (up to $35 \%$ ) in one of the series of malignant transformation in ameloblastoma. ${ }^{9}$ Distant metastases to lung $(70-85 \%$ of total cases), followed by bone, liver, and brain. ${ }^{9}$ Metastasis occurs via lymphatic (rare) or hematogenous spread. As per the recent studies, two central pathways, Mitogen Activated Protein Kinases (MAPK) and sonic hedgehog $(\mathrm{SHH})$, were identified play key roles in ameloblastic oncogenesis. ${ }^{9}$
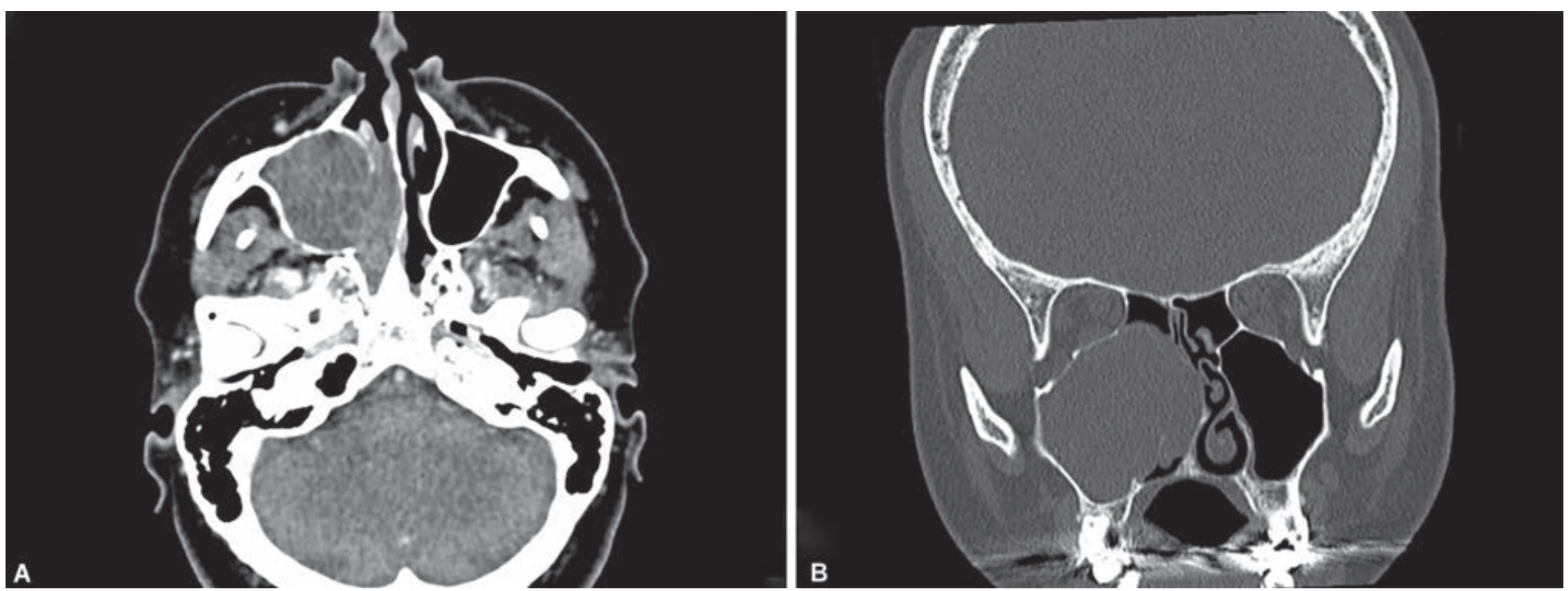

Figs 2A and B: Contrast-enhanced computed tomography of paranasal sinus depicting lobulated nonenhancing soft tissue in right maxillary sinus extending posteriorly through right posterior choana into nasopharynx: suggestive of right antrochoanal polyp: (A) Axial view slice thickness: $0.3 \mathrm{~mm}$; (B) Coronal view: slice thickness: $0.3 \mathrm{~mm}$ 

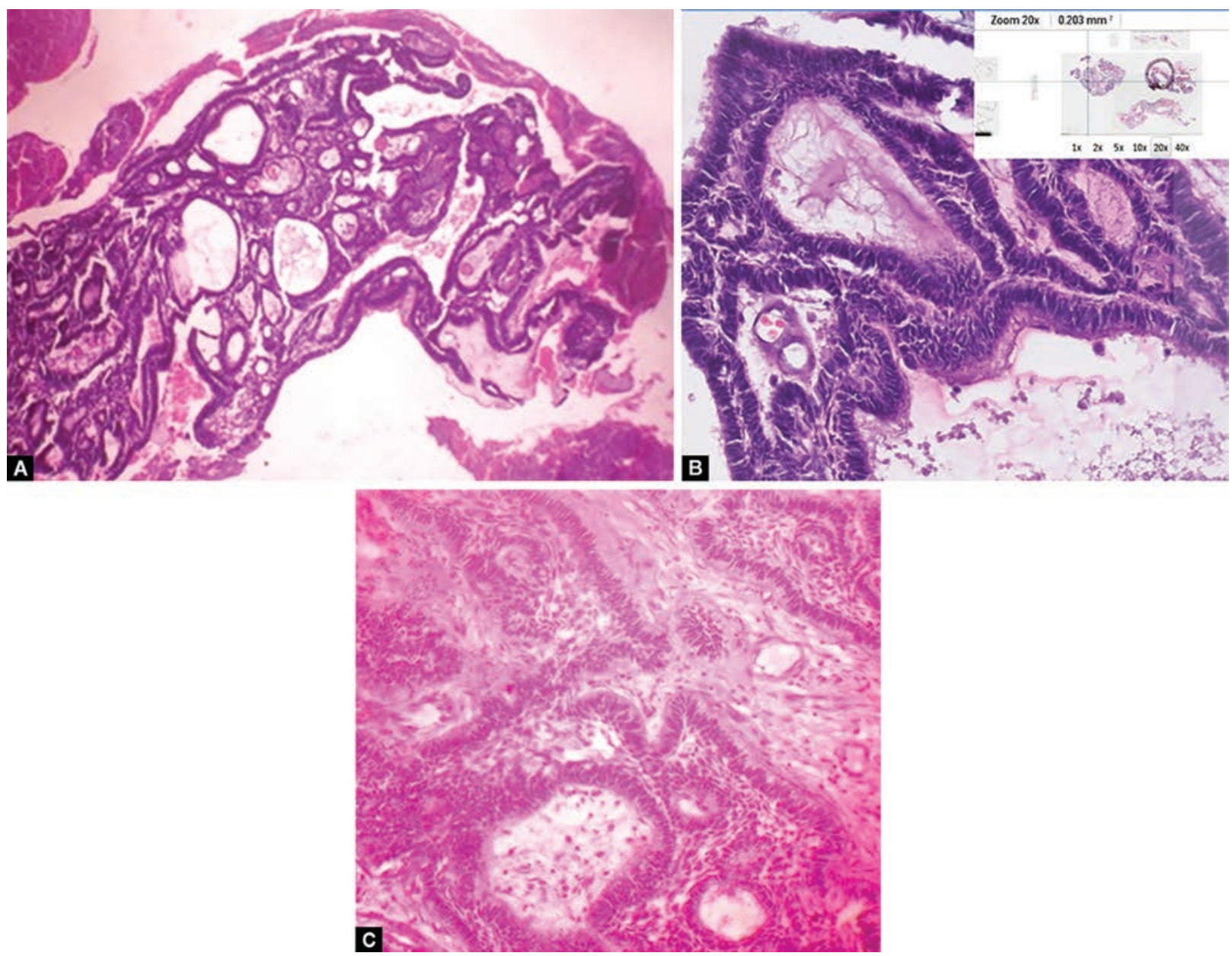

Figs 3A to C: Hematoxyline and eosin stain: Microphotograph of histological appearance of tissue depicting odontogenic epithelial islands in plexiform and follicular pattern: (A) Scanner view; (B) Magnification at 20x; (C) Magnification at 40x)

The treatment consists of en-bloc resection of tumor with $1-2 \mathrm{~cm}$ bone margins and immediate bone reconstruction for restoring form and functions, that is, speech and swallowing. Margins are required as microscopic tumor extension of 2-8 $\mathrm{mm}$ (mean of $4.5 \mathrm{~mm}$ ) beyond the radiographic boundaries of the tumor has been reported in ameloblastoma. ${ }^{9}$ Recommended margins are $1-1.5 \mathrm{~cm}$ for unicystic, $1.5-2 \mathrm{~cm}$ for solid/multicystic, and around $2-3 \mathrm{~cm}$ for ameloblastic carcinoma. ${ }^{9}$

Radiotherapy have been attempted in recurrent or inoperable cases; however, its efficacy is still unclear. ${ }^{9}$ Reports on systemic chemotherapy suggested that ameloblastoma may be sensitive to platinum-based agents, and chemotherapy may have a role in the improvement of clinical symptoms in nonsurgical patients.

Prognosis for ameloblastoma depends on the patient's age, tumor size, extent of disease, location of tumor, and histological type. Overall recurrence rate is $33 \%$ : lesser with radical surgery (021\%) more with conservative resections (33-96\%). ${ }^{9}$ Case reports suggested metastatic ameloblastoma to the lungs associated with a paraneoplastic syndrome causing hypercalcemia. ${ }^{9}$

For metastatic ameloblastoma, median disease-free interval (DFI) of 13 years and mean survival up to 2 years have been described. $^{10}$

Hasim et al. reported longest survival after diagnosis of metastases (37 years) in a patient with bilateral pulmonary metastases, who remained asymptomatic and did not require any treatment. 10

Maxilla is relatively rare subsite for ameloblastoma that too more often the epicenter is in the alveolar process of maxilla. However, in the present case the epicenter was from maxillary antrum and not at all from alveolar process.

Thyroid hormone has been known to be playing a role in bone metabolism. An increased level of TSH in blood stimulates increased bone formation. Hypothyroidism in the present case was incidentally detected on blood investigations. However, in view of recent case reports coincidence of both hypothyroidism and ameloblastoma have been observed in certain cases of ameloblastoma. ${ }^{11}$ There is further room for research in this aspect. Although ameloblastoma is developed from dental epithelial remnants, the presence of ameloblastoma in maxilla with epicenter from maxillary antrum and not at all from alveolar process is a rare phenomena. Probably this is the first case the first case of its kind of unusual occurrence of maxillary ameloblastoma with epicenter from maxillary antrum and not at all from alveolar process, unusual presentation as presenting symptom being only dry cough (without any other nasal symptoms such as nasal blockage, facial fullness, facial heaviness, headache, nasal bleeding, etc.) and antrochoanal polyp been detected incidentally. The diagnosis would have been missed in 


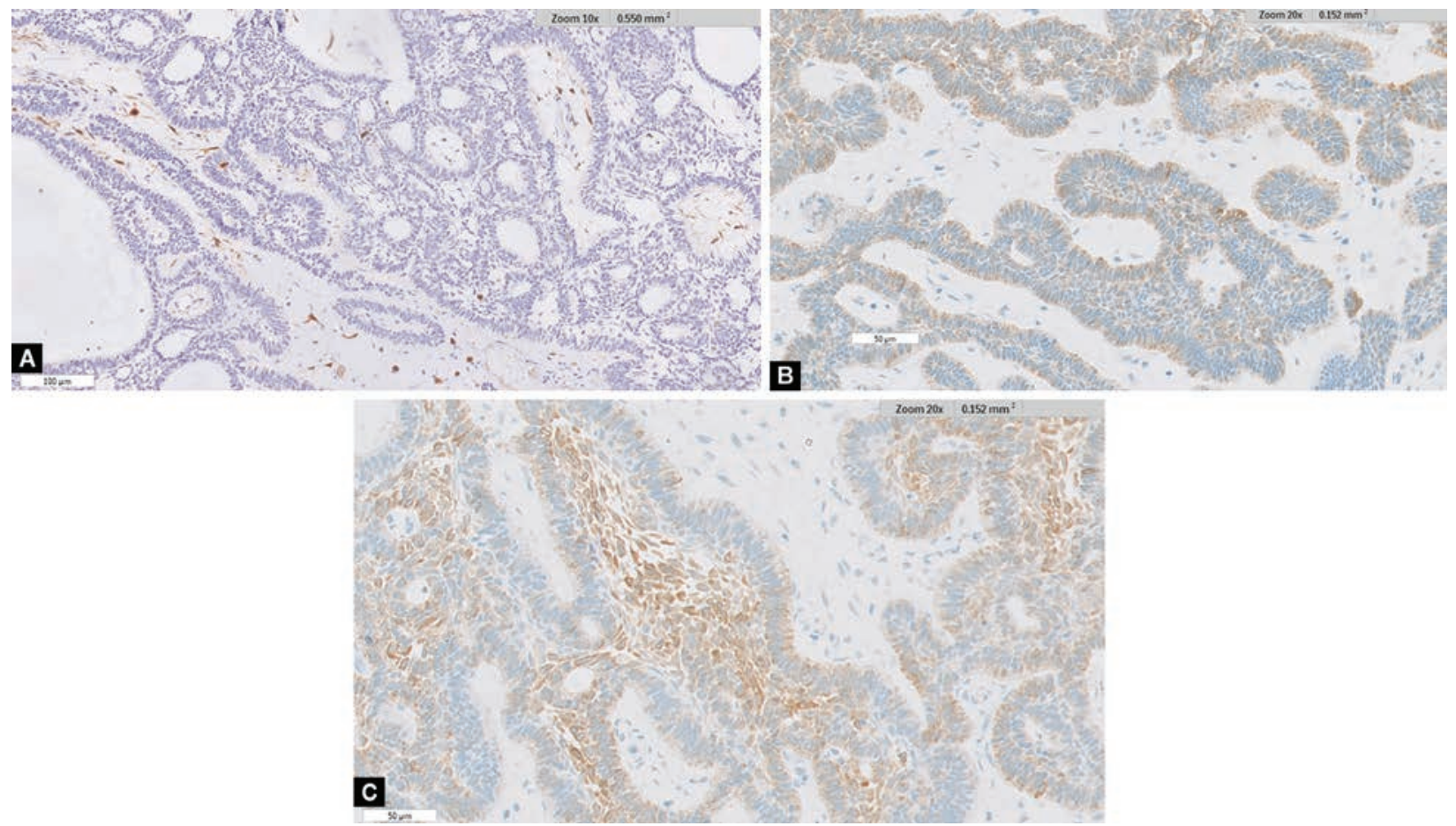

Figs 4A to C: (A) Photomicrograph of immunohistochemistry slide showing positivity to calretinin in stellate reticulin like cells (magnification: 10X); (B) Photomicrograph of immunohistochemistry slide showing positivity to CK14 in dentine epithelium (magnification: 20X); (C) Photomicrograph of immunohistochemistry slide showing positivity to CK19 in dentine epithelium (magnification: 20X)
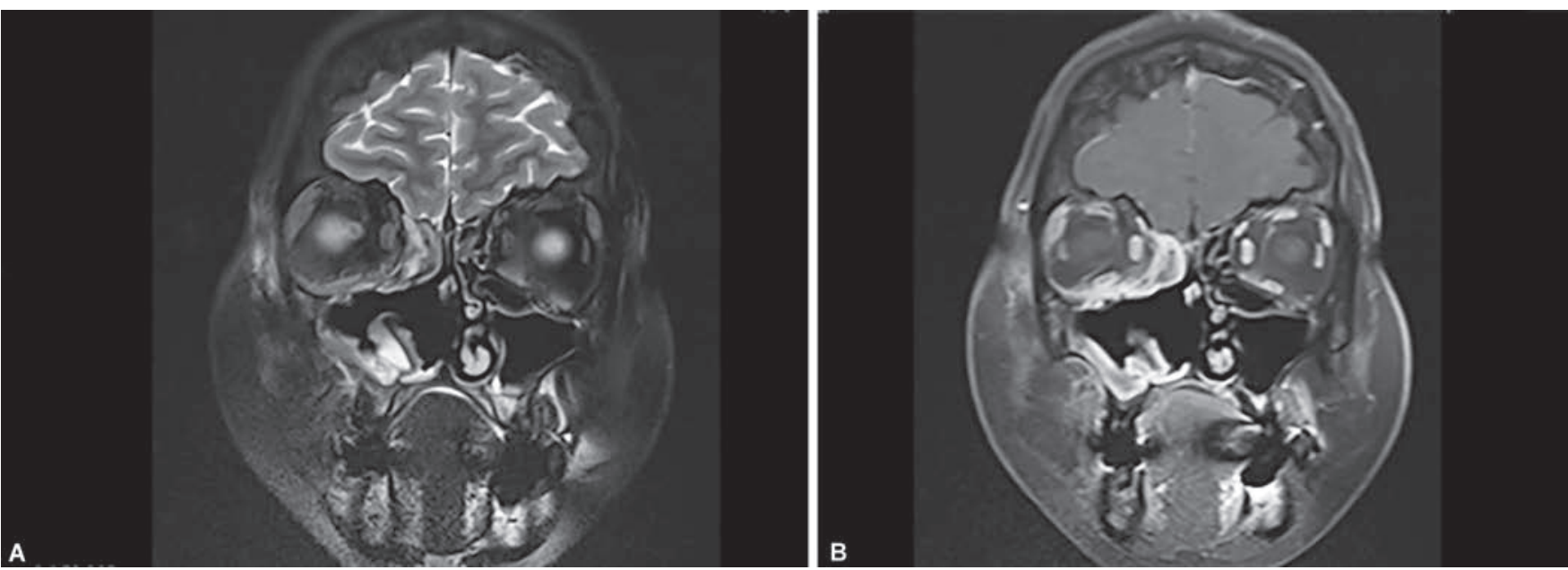

Figs 5A and B: MRI paranasal sinuses with contrast depicting contrast-enhancing soft tissue in the floor of right maxillary sinus along with right ethmoid and frontal sinus (A: axial view; B: coronal view $T 1$ with contrast slice thickness: $3 \mathrm{~mm}$ )

this case at early stage without meticulous histology follow-up and the disease would have then be detected to much later stage after the development of symptoms, which then would require greater resection and reconstruction on skin involvement. This occurrence of the ameloblastoma from maxillary antrum may suggest the possibility of entrapment of dental epithelium in maxillary antrum during maxillary development.

\section{Conclusion}

Maxillary ameloblastoma is a rare in occurrence. Although embryologically derived from remnant of dental epithelium, it may arise entirely from maxillary antrum as in this case and may present as antrochoanal polyp without any specific symptoms. The coincidence of hypothyroidism in this case may be a contributory factor as the thyroid hormone plays role in bone metabolism which needs to be further studied in this aspects. Thus to conclude, antrochoanal polyp may be the early presenting feature of ameloblastoma of maxilla and hence needs to be taken into consideration as one of the differential diagnosis. As such it is imperative for its meticulous histological review for early detection and subsequent management by appropriate en-bloc resection, further punctilious histology and careful follow-up are required. 

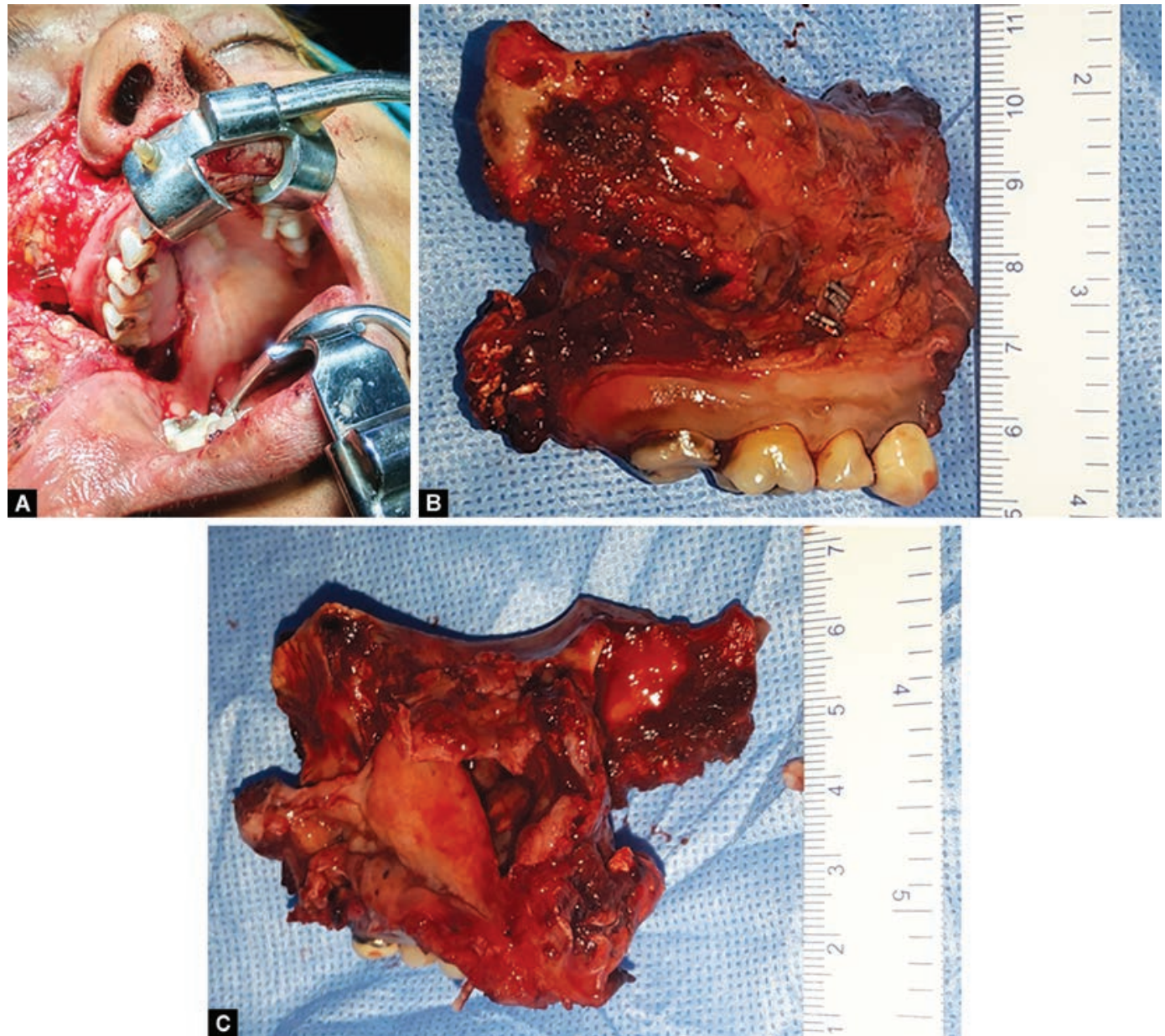

Figs 6 A to C: (A) Intraoperative photograph during right total maxillectomy; (B) Clinical photograph of specimen post "en-bloc" resection; (C) Clinical photograph of specimen post "en-bloc" resection

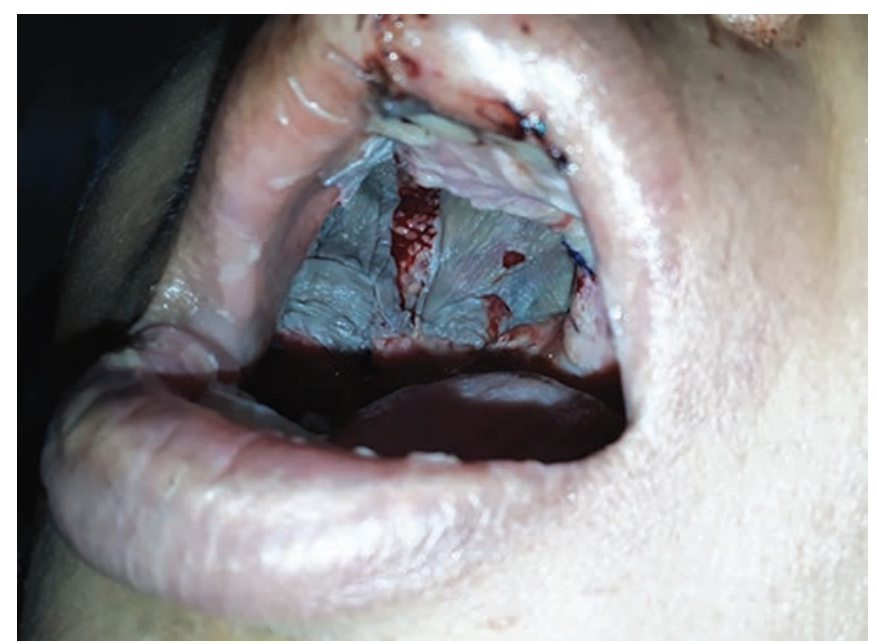

Fig. 7: Clinical photograph depicting healing cavity at 1 week postoperatively

\section{References}

1. Cusack JW. Report of the amputations of the lower jaw. Dubliln. Hop Rec 1827:4:1-38. DOI: 10.17826/cutf.324546

2. Ivery RH, Churchill HR. The need of a standardized surgical and pathological classification of tumors and anomalies of dental origin. Am Assoc Dent Sch Trans 1930;7:240-245.
3. Brazis PW, Miller NR, Lee AG, et al. Neuroophthalmologic aspects of ameloblastoma. Skull Base Surg 1995;5(4):233-244. DOI: 10.1055/s-2008-1058921

4. Reichart PA, Philipsen HP, Sonner S. Ameloblastoma: biological profile of 3677 cases. Eur J Cancer B Oral Oncol 1995;31B(2):86-99. DOI: 10.1016/0964-1955(94)00037-5

5. Schafer DR, Thompson LD, Smith BC, et al. Primary ameloblastoma of the sinonasal tract: a clinicopathologic study of 24 cases. Cancer 1998;82(4):667-674. DOI: 10.1002/(sici)1097-0142(19980215) 82:4<667::aid-cncr8>3.0.co;2-i

6. Wenig BM. Atlas of Head and Neck Pathology, 2nd edn. Elsevier Saunders; 2007.

7. Olaitan AA, Adeola DS, Adekeye EO. Ameloblastoma: clinical features and management of 315 cases from Kaduna, Nigeria.J Craniomaxillofac Surg 1993;21(8):351-355. DOI: 10.1016/s1010-5182(05)80497-4

8. Singer SR, Mupparapu M, Philipone E. Cone beam computed tomography findings in a case of plexiform ameloblastoma. Quintessence Int 2009;40(8):627-630. PMID:19639086.

9. McClary AC, West RB, McClary AC, et al. Ameloblastoma: a clinical review and trends in management. Eur Arch Otorhinolaryngol 2016;273(7):1649-1661. DOI: 10.1007/s00405-015-3631-8

10. Hasim FW, Poon CC, Smith AC. Prolonged survival with confirmed metastatic pulmonary ameloblastoma. Int J Oral Maxillofac Surg 2007;36:953-955. DOI: 10.1016/j.ijom.2007.02.010

11. Okade D, Nagaraj T, Sumana CK, et al. Desmoplastic ameloblastoma of anterior mandible: a rare case report. J Med Radiol Pathol Surg 2016;3:16-18. DOI: 10.15713/ins.jmrps.76 